\title{
A NEAR-INFRARED SPECTROSCOPIC STUDY OF THE ACCRETING MAGNETIC WHITE DWARF SDSS J121209.31+013627.7 AND ITS SUBSTELLAR COMPANION
}

\author{
J. FARIHI, ${ }^{1,2}$ M. R. BURLeigh, ${ }^{3}$ AND D. W. HOARD ${ }^{4}$ \\ Received 2007 August 7; accepted 2007 October 27
}

\begin{abstract}
The nature of the excess near-infrared emission associated with the magnetic white dwarf commonly known as SDSS 1212 is investigated primarily through spectroscopy, and also via photometry. The inferred low-mass secondary in this system has been previously detected by the emission and variation of $\mathrm{H} \alpha$, and the $1-2.5 \mu \mathrm{m}$ spectral data presented here are consistent with the presence of a late $\mathrm{L}$ or early $\mathrm{T}$ dwarf. The excess flux seen beyond $1.5 \mu \mathrm{m}$ in the phaseaveraged spectrum is adequately modeled with an L8 dwarf substellar companion and cyclotron emission in a $7 \mathrm{MG}$ magnetic field. This interesting system manifests several observational properties typical of polars, and is most likely an old interacting binary with a magnetic white dwarf and a substellar donor in an extended low state.
\end{abstract}

Subject headings: binaries: close — infrared: stars — novae, cataclysmic variables — stars: evolution — stars: formation — stars: fundamental parameters — stars: individual (SDSS J121209.31+013627.7) — stars: low-mass, brown dwarfs — white dwarfs

\section{INTRODUCTION}

Cataclysmic variables are spectrally exotic binaries, displaying a diversity and complexity of features, behavior, and variability, effectively masking the spectral signatures of their coolest secondaries. Despite unyielding efforts to unambiguously and directly detect likely substellar companions in either nonmagnetic or magnetic cataclysmic variables, these altered degenerate dwarfs remain largely elusive (Littlefair et al. 2003). The mass determination of the substellar donors in two nonmagnetic accreting binaries reported by Littlefair et al. $(2006,2007)$ remain rare among cataclysmic variables, although the determination of their substellarity was indirect. Cataclysmic variables containing strongly magnetic white dwarfs (called polars because of the highly polarized nature of their light) offer a slight advantage by lacking the often overwhelming luminosity from an accretion disk. On the other hand, their magnetic fields generate copious cyclotron radiation (in addition to flux from direct accretion), which is not present in nonmagnetic cataclysmic variables.

Polars are also known for exhibiting long periods of quiescence in which many secondaries have been directly observed, although none of spectral type later than M (Harrison et al. 2005). The recently recognized class of low accretion rate polars may provide decent infrared hunting grounds for substellar companions, if present, as their cyclotron harmonics appear mainly in the optical due to $B \sim 50 \mathrm{MG}$ fields. It has been suggested that no mass transfer takes place in these systems, but rather the efficient capture of secondary wind, and that their separations are consistent with being essentially detached (Schmidt et al. 2005b). Yet, it is difficult to certify that these binaries are not simply in protracted low states such as seen in other polars (Schwope et al. 2007; Harrison et al. 2004).

\footnotetext{
1 Gemini Observatory, Northern Operations, 670 North A‘ohoku Place, Hilo, HI 96720 .

2 Department of Physics and Astronomy, University of California, 430 Portola Plaza, Los Angeles, CA 90095; jfarihi@astro.ucla.edu.

3 Department of Physics and Astronomy, University of Leicester, Leicester LE1 7RH, UK; mbu@star.le.ac.uk.

4 Spitzer Science Center, California Institute of Technology, MS 220-6, Pasadena, CA 91125; hoard@ipac.caltech.edu.
}

Recent Spitzer IRAC observations of several polars indicate flat infrared spectral energy distributions which are difficult to reconcile with cool degenerate companions alone, and may indicate circumbinary dust as well as underlying cyclotron continuum emission (Brinkworth et al. 2007; Howell et al. 2006). It will be interesting to see the results of similar mid-infrared observations with a larger sample of polars as well as observations at longer wavelengths where there should be little or no cyclotron emission even in $B \approx 10 \mathrm{MG}$ fields. The first published Spitzer IRS spectrum of a polar strongly supports the presence of circumbinary dust at EF Eridani (Hoard et al. 2007a).

This paper represents a spectroscopic effort to directly detect the almost certain substellar companion to the cool magnetic white dwarf SDSS J121209.31+013627.7 (SDSS 1212). Its binarity was reported with optical spectra supplemented with a single $J$-band photometric observation, limiting the spectral type of the secondary to L5 or later, and was initially thought to represent a detached system (Schmidt et al. 2005a). This possibility was still considered viable despite a second study which detected strong cyclotron emission - a likely indicator of mass transferin $K$-band time series photometry (Debes et al. 2006). Both Koen \& Maxted (2006) and Burleigh et al. (2006a) detected optical variability and a light curve similar to that shown by polars in low states. Burleigh et al. (2006a) further showed that the amplitude of the modulations was larger at blue and ultraviolet wavelengths than in the red, consistent with a hot accretion spot responsible for the cyclotron emission. Burleigh et al. (2006a) also detected X-ray emission from SDSS 1212, conclusively proving accretion is taking place. This paper presents cross-dispersed 1-2.5 $\mu \mathrm{m}$ spectroscopy and spectral modeling, along with $H K$ photometry, in order to constrain the source(s) of the observed near-infrared excess emission.

\section{OBSERVATIONS}

\subsection{HK Photometry}

Near-infrared images of SDSS 1212 were acquired on 2006 June 10 at the United Kingdom Infrared Telescope under photometric conditions $(\sec z<1.1$ ) with the Wide Field Camera (WFCAM; M. M. Casali et al. 2008, in preparation; Hirst et al. 2006; Henry et al. 2003). A 5-point dither pattern was used with 
TABLE 1

WFCAM Рнотometric Calibration Data

\begin{tabular}{|c|c|c|c|c|c|c|}
\hline Number & 2MASS Name & $\begin{array}{c}H \\
(\mathrm{mag})\end{array}$ & $\begin{array}{c}K_{s} \\
(\mathrm{mag})\end{array}$ & $\begin{array}{c}H_{0}{ }^{\mathrm{a}} \\
(\mathrm{mag})\end{array}$ & $\begin{array}{c}K_{0}^{\mathrm{a}} \\
(\mathrm{mag})\end{array}$ & Remarks \\
\hline $1 \ldots \ldots \ldots \ldots \ldots \ldots \ldots$ & $\mathrm{J} 12114360+0137020$ & $13.841 \pm 0.035$ & $13.701 \pm 0.050$ & 23.032 & 22.354 & $\ldots$ \\
\hline $2 \ldots \ldots \ldots \ldots \ldots \ldots \ldots$ & $\mathrm{J} 12114583+0139374$ & $13.435 \pm 0.030$ & $13.337 \pm 0.038$ & 23.083 & 22.391 & $\ldots$ \\
\hline $3 \ldots \ldots \ldots \ldots \ldots \ldots \ldots$ & $\mathrm{J} 12114736+0133594$ & $14.098 \pm 0.039$ & $14.105 \pm 0.063$ & 23.031 & 22.426 & $\ldots$ \\
\hline ......... & $\mathrm{J} 12114758+0137223$ & $14.021 \pm 0.037$ & $14.062 \pm 0.062$ & 23.040 & 22.479 & $\ldots$ \\
\hline $5 \ldots \ldots \ldots \ldots \ldots \ldots \ldots$ & $\mathrm{J} 12115403+0132049$ & $13.991 \pm 0.046$ & $13.766 \pm 0.058$ & 23.069 & 22.325 & $\ldots$ \\
\hline $6 \ldots \ldots \ldots \ldots \ldots \ldots \ldots$ & $\mathrm{J} 12115541+0130399$ & $13.431 \pm 0.027$ & $13.299 \pm 0.035$ & 23.041 & 22.356 & $\ldots$ \\
\hline $7 \ldots \ldots \ldots \ldots \ldots \ldots$ & $\mathrm{J} 12120408+0135365$ & $15.046 \pm 0.066$ & $14.776 \pm 0.123$ & 23.076 & 22.282 & 1,2 \\
\hline $8 \ldots \ldots \ldots \ldots \ldots \ldots \ldots$ & $\mathrm{J} 12120582+0135157$ & $14.987 \pm 0.072$ & $14.955 \pm 0.138$ & 22.980 & 22.404 & 1,2 \\
\hline $9 \ldots \ldots \ldots \ldots \ldots \ldots \ldots$ & $\mathrm{J} 12120662+0138153$ & $14.065 \pm 0.053$ & $13.974 \pm 0.068$ & 23.075 & 22.405 & $\ldots$ \\
\hline $10 \ldots \ldots \ldots \ldots \ldots \ldots$ & $\mathrm{J} 12120989+0135259$ & $15.894 \pm 0.174$ & $15.538 \pm 0.232$ & 22.908 & 22.115 & 1,2 \\
\hline $11 \ldots \ldots \ldots \ldots \ldots \ldots$ & $\mathrm{J} 12121658+0137042$ & $14.290 \pm 0.041$ & $14.137 \pm 0.066$ & 23.039 & 22.452 & 2 \\
\hline $12 \ldots \ldots \ldots \ldots \ldots \ldots$ & $\mathrm{J} 12121667+0135257$ & $13.868 \pm 0.022$ & $13.805 \pm 0.068$ & 23.036 & 22.383 & 2 \\
\hline $13 \ldots \ldots \ldots \ldots \ldots \ldots$ & $\mathrm{J} 12122238+0130101$ & $12.927 \pm 0.022$ & $12.904 \pm 0.032$ & 23.058 & 22.410 & $\ldots$ \\
\hline $14 \ldots \ldots \ldots \ldots \ldots \ldots$ & $\mathrm{J} 12122266+0137425$ & $13.602 \pm 0.041$ & $13.536 \pm 0.055$ & 23.011 & 22.470 & $\cdots$ \\
\hline $15 \ldots \ldots \ldots \ldots \ldots \ldots$ & $\mathrm{J} 12122647+0140477$ & $13.040 \pm 0.029$ & $13.030 \pm 0.037$ & 23.070 & 22.412 & $\ldots$ \\
\hline
\end{tabular}

Notes.-(1) Not used in the present paper. (2) Used in Debes et al. (2006).

a Transformations between 2MASS and UKIRT photometric systems were ignored (see $\S 3.1$ ).

$2 \times 10 \mathrm{~s}$ exposures at each position; this pattern was repeated twice at $H$ and thrice at $K$ for total exposure times of 200 and $300 \mathrm{~s}$, respectively. The data were reduced in the standard fashion. Each stack was median combined to extract a sky frame which was also normalized to generate a flat-field map. Individual skysubtracted, flat-fielded frames were registered and averaged for a final frame on which to execute measurements. The full widths at half-maximum of the reduced stellar images were 2.0 and 1.9 WFCAM pixels $\left(0.40^{\prime \prime}\right.$ pixel $\left.^{-1}\right)$ at $H$ and $K$, respectively. Aperture photometry was performed with standard IRAF tasks using an $r=1.20^{\prime \prime}$ aperture and an $r=8^{\prime \prime}-12^{\prime \prime}$ sky annulus.

Due to the very wide field of view of WFCAM $\left(819^{\prime \prime} \times 819^{\prime \prime}\right)$, 15 Two Micron All Sky Survey (2MASS) point sources in the same field as SDSS 1212 were simultaneously imaged to choose among as possible photometric calibrators. Each 2MASS star chosen as a calibrator had all of the following properties: a full width at half-maximum consistent with linear accumulation (i.e., unsaturated), high signal-to-noise ratios $(\mathrm{S} / \mathrm{Ns}>100)$ in the reduced WFCAM images, and reliable 2MASS photometry at both wavelengths $\left(\sigma_{H}<3.4 \%, \sigma_{K_{s}}<6.3 \%\right.$; Skrutskie et al. 2006). The selected 12 calibrators are listed in Table 1, along with their 2MASS data and their derived instrumental zero points.

Additional photometry was acquired on 2006 July 3 at the Gemini Telescope North under photometric conditions with the Near-Infrared Imager (NIRI; Hodapp et al. 2003). Both SDSS 1212 and the United Kingdom Infrared Telescope (UKIRT) standard star FS 132 (Hawarden et al. 2001) were observed in $H K$ at nearly identical average air masses of $\sec z \approx 1.95$, yielding extinction corrections less than 0.003 mag. A 5-point dither pattern was used for both science target and calibrator, with an extra frame taken for SDSS 1212. The calibrator was observed $10 \times 1 \mathrm{~s}$ at each position while the science target was observed $3 \times 12 \mathrm{~s}$ at each position, yielding total exposure times of $50 \mathrm{~s}$ for FS 132 and $216 \mathrm{~s}$ for SDSS 1212 at $H K$. The data were reduced in a manner similar to the WFCAM data, with separately acquired and processed flat-field images. The full widths at half-maximum of the reduced stellar images were between 4.5 and 4.9 NIRI pixels $\left(0.12^{\prime \prime}\right.$ pixel $^{-1}$ at f/6). Aperture photometry was performed similarly using an $r=0.47^{\prime \prime}$ aperture and an $r=2.3^{\prime \prime}-3.5^{\prime \prime}$ sky annulus.
The photometry results are listed in Table 2 and plotted in Figure 1. Column (3) in the table gives the uncertainty in the measured aperture photometry (due to intrinsic $\mathrm{S} / \mathrm{N}$ limitations). Column (4) in the table gives the calibration uncertainty: for the WFCAM data, this is the standard deviation in the derived zero points; for the NIRI data, an intrinsic 3\% error was assumed.

\subsection{1-2.5 $\mu \mathrm{m}$ Spectroscopy}

Spectroscopy was performed on 2006 June 19 and 23 at the Gemini Telescope South with the Near-Infrared Spectrograph (GNIRS; Elias et al. 1998). Observations were executed in crossdispersed mode with the $31.71 \mathrm{~mm}^{-1}$ grating and the $0.3^{\prime \prime}$ wide slit, yielding $R \approx 1700$ while effectively covering the entire nearinfrared range without interorder contamination. Data were taken at two nod positions along the $6^{\prime \prime}$ long slit, with $300 \mathrm{~s}$ exposures at each nod position, repeated six times for a total on-source integration time of $1 \mathrm{hr}$ on each of two nights. The telluric standard HIP 60030 (A7 V, $V-K=0.5$ ) was observed in a similar manner with $6 \mathrm{~s}$ exposures at each nod position, repeated four times.

The raw data were processed with standard GNIRS tools within the Gemini IRAF package version 1.9. These steps included flatfield and pinhole map creation from calibration lamps, bad pixel correction, linearity correction, sky subtraction, image shifting and averaging, order cutting, spatial rectification, and wavelength calibration from argon lamps. Spectral extraction of each order was performed with a GNIRS equivalent to the IRAF apall task.

TABLE 2

Photometry of SDSS 1212

\begin{tabular}{|c|c|c|c|c|c|}
\hline $\begin{array}{l}\text { Passband } \\
\text { (1) }\end{array}$ & $\begin{array}{c}\text { Instrument } \\
\text { (2) }\end{array}$ & $\begin{array}{c}\text { Magnitude } \\
\text { (mag) } \\
(3)\end{array}$ & $\begin{array}{c}\sigma_{\text {phot }} \\
\text { (mag) } \\
(4)\end{array}$ & $\begin{array}{c}\sigma_{\mathrm{cal}} \\
(\mathrm{mag}) \\
(5)\end{array}$ & $\begin{array}{c}\sigma_{\text {total }} \\
(\mathrm{mag}) \\
(6)\end{array}$ \\
\hline .................... & WFCAM & 17.676 & 0.089 & 0.021 & 0.091 \\
\hline 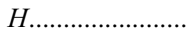 & NIRI & 17.710 & 0.064 & 0.030 & 0.071 \\
\hline 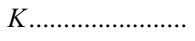 & WFCAM & 17.372 & 0.105 & 0.047 & 0.114 \\
\hline К & NIRI & 16.693 & 0.033 & 0.030 & 0.045 \\
\hline
\end{tabular}

Notes.-An intrinsic calibration uncertainty of $3 \%$ was assumed for the NIRI data. See Fig. 1 for the phases corresponding to the photometric observations. 


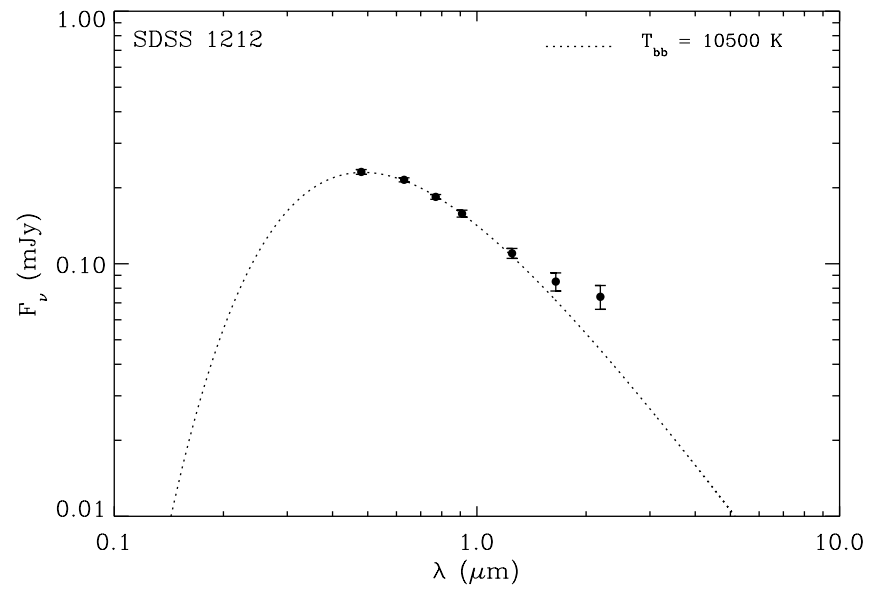

FIG. 1.- Spectral energy distribution of SDSS 1212. Optical gri and nearinfrared $z J$ photometry is from Schmidt et al. (2005a). The $H$-band value is the average of the NIRI and WFCAM measurements, while the $K$-band value is the flux measured during minimum (WFCAM), all from the present paper (Table 2). The grizJ photometric fluxes are well reproduced by a 10,500 K blackbody.

Some processing of the raw data was required prior to application of the standard package tools listed above. The first and second science frames on each night contained significant residual signal (between 3 and 5 times larger than the read level in the unexposed portion of the array) from the target acquisition mirror. The raw acquisition images were scaled to match the contamination level of the affected frames and subtracted off, effectively solving this problem. In addition, there is an occasionally occurring bias level problem in one quadrant of the detector which appeared in many of the science exposures. This noise was well removed with the nvnoise task within the GNIRS tools.

Both the telluric standard and SDSS 1212 were processed in an identical fashion until the spectral extraction step. Instead of the $8-10$ pixel diameter $\left(1.20^{\prime \prime}-1.50^{\prime \prime}\right)$ aperture used for HIP 60030 , a $4-5$ pixel diameter $\left(0.60^{\prime \prime}-0.75^{\prime \prime}\right)$ aperture was used for SDSS 1212 to maximize the extracted spectroscopic $\mathrm{S} / \mathrm{N}$ of this faint source. Flux calibration and telluric correction were performed for each order with Spextool (ver. 3.3; Cushing et al. 2004; Vacca et al. 2003), using the general xtellcor package. The flux levels of all four spectral orders presented here agree quite well at their boundaries, and hence no interorder adjustment was necessary. However, the entire cross-dispersed spectrum, the average of both nights' data, was adjusted slightly by a single scaling factor to best match the $J$ - and $H$-band photometry in Figure 1. This unsmoothed spectrum is displayed in Figure 2 together with the $J H K$ points from Figure 1.

\section{RESULTS}

When telescope time was requested for this study, it was not yet known that SDSS 1212 was variable in any broad wavelength bands. Apart from the $\mathrm{H} \alpha$ line strength variability reported by Schmidt et al. (2005a), SDSS 1212 is now known to be variable in $u g i, V R$ (and unfiltered white optical light), and $K$, but not in $J H$ (Burleigh et al. 2006a; Koen \& Maxted 2006; Debes et al. 2006).

Figure 3 shows the corresponding phase of SDSS 1212 for all the photometric and spectroscopic observations. The WFCAM photometry was taken during $K$-band minimum, by which it is meant that all the $2.2 \mu \mathrm{m}$ flux should be near its minimum valuewhether this minimum flux comes from cyclotron continuum emission, the substellar companion, or a combination of sources is uncertain at present and the focus of this study. The NIRI pho-

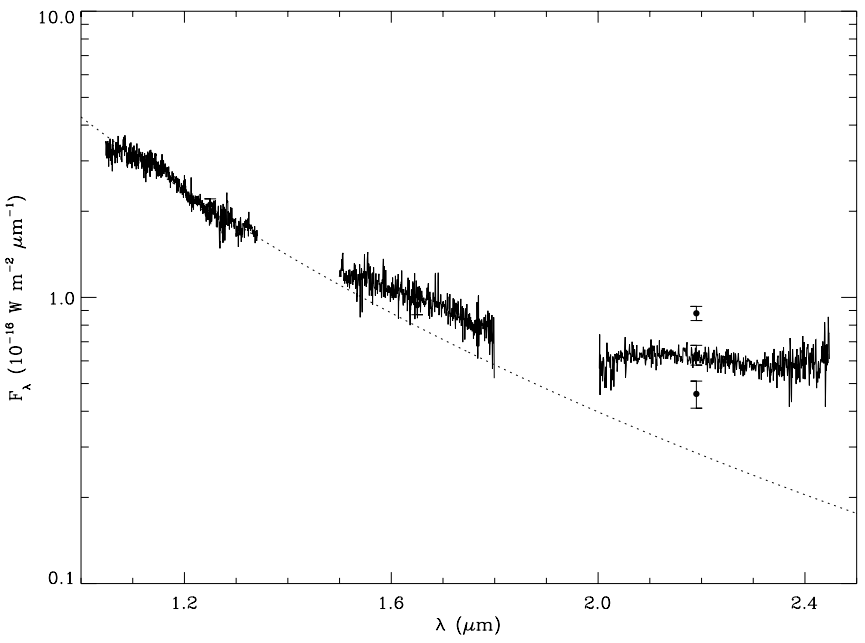

FIG. 2.-Flux-calibrated GNIRS spectrum of SDSS 1212, averaged over all nights and phases. Four cross-dispersed orders are displayed within the atmospheric windows. The photometric points and blackbody continuum are identical to that plotted in Fig. 1, with two additional (calculated) $K$-band flux points described in $\S 3.3$.

tometry, on the other hand, was taken close to the $K$-band maximum, which occurs near orbital phases $\phi=0.8$ and 0.2 , but not at $\phi=0.0$ as for the optical and ultraviolet peak (see $\S 3.2$ ).

\subsection{WFCAM Photometry during Minimum}

It is somewhat surprising that the nominal WFCAM $H$ and $K$ magnitudes in Table 2 are $0.12-0.15$ mag fainter than the minimum reported by Debes et al. (2006), although the data sets have overlapping error bars at each passband. As can be seen in Figure 3, it has been verified that the WFCAM observations were taken during the photometric minimum by comparing the corresponding corrected MJD with the ephemeris of Burleigh et al. (2006a).

Five of the stars listed in Table 1 were used as calibrators by Debes et al. (2006). However, the 2MASS photometric errors on three of these objects, while mainly decent at $J$, become relatively high at $H$ and $K_{S}\left(\sigma_{J}<11 \%, \sigma_{H}<17 \%, \sigma_{K_{s}}<25 \%\right)$, and were

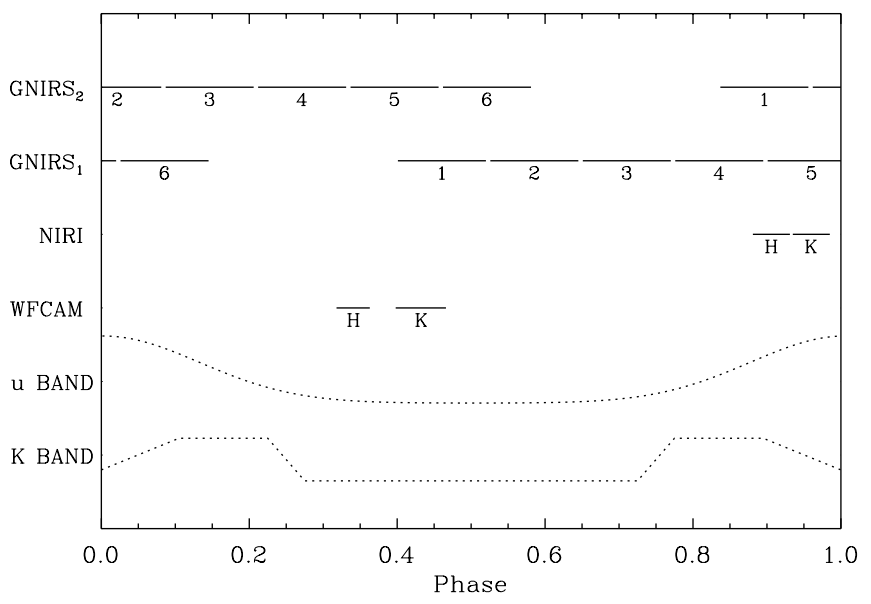

FIG. 3.-Orbital phases corresponding to the photometric and spectroscopic observations with each instrument: GNIRS, NIRI, and WFCAM. The numbers 1-6 denote each of six nodded pairs of GNIRS observations taken on two separate nights (subscripted 1 and 2). Also shown are the $u$-band light-curve model fit of Burleigh et al. (2006a) and the rough $K$-band light behavior (Debes et al. 2006), both normalized to 1 . The relative amplitude of the $u$-band peak has been exaggerated by a factor of 10 for visibility. 
discarded here for this reason. In order to investigate whether this fact could account for the discrepancy between the reported $H K$ magnitudes - and more importantly, to assess the likelihood that this difference is real- the instrumental zero points for the three discarded calibrators were compared with those derived with the 12 higher $\mathrm{S} / \mathrm{N}$ stars used here. Those three stars alone yield an $H_{0}$ and $K_{0}$ which differ by 3 standard deviations $(-0.06$ mag at $H$, -0.14 mag at $K$ ) from the mean zero points used for the Table 2 WFCAM results. In addition, according to Debes et al. (2006) not all five of these calibrators were present in every frame of their WIRC and PANIC data. The three stars with lowest S/N (those discarded here) are in fact closest on the sky to SDSS 1212, at $61^{\prime \prime}-93^{\prime \prime}$, while the remaining two calibrators are $115^{\prime \prime}-126^{\prime \prime}$ distant. Hence the three lower quality 2MASS calibrators had a good chance of being present in most, if not all, of the WIRC frames (a single chip is $201^{\prime \prime} \times 201^{\prime \prime}$ ), while the two higher quality calibrator stars were unlikely to be imaged in the majority of that data set. Owing to the smaller field of view of PANIC $\left(128^{\prime \prime} \times 128^{\prime \prime}\right)$, only the three lowest S/N 2MASS calibrators were used (Debes et al. 2006).

There are two additional reasons why the WFCAM magnitudes in Table 2 may be slightly discrepant from the previously published values. First, neither study has transformed the photometry from the 2MASS system into the native filter system through which the observations were taken. In the present case, any errors introduced by failing to transform the photometry are much smaller than the errors due to the intrinsic $\mathrm{S} / \mathrm{N}$ (represented by $\sigma_{\text {phot }}$ in Table 2). Also, the fact that 12 calibrators were used here would almost certainly shrink any error of this type even further due to averaging (Carpenter 2001). Second, it is conceivable that SDSS 1212 exhibits further variability, which has so far not been documented.

\subsection{NIRI Photometry near Maximum}

The NIRI photometry is also listed in Table 2 . It has been verified that these observations were taken near the minimum hot spot viewing angle. Although this corresponds to a maximum in ultraviolet and optical light, it does not quite correspond to the cyclotron maximum as seen in the $K$-band light curve measured by Debes et al. (2006), which occurs when the hot spot is on either limb-immediately before and after the ultraviolet maximum $(\phi=0.0$ in this paper and Burleigh et al. 2006a; $\phi=0.5$ in Debes et al. 2006).

\subsection{GNIRS Phase-averaged Spectroscopy}

The spectroscopy essentially covers all orbital phases of SDSS 1212 because the flux was sufficiently low $(0.1 \mathrm{mJy}$ at $1.6 \mu \mathrm{m})$ as to preclude extracting phase-resolved data - the observation itself represents a benchmark near the limit of what is possible with the most advanced ground-based telescopes and instruments. The average of all data from both nights yields $\mathrm{S} / \mathrm{Ns}$ in the range 1017 (average of 14) at $H$ and 10-26 (average of 18) at $K$, estimated using 12 sections of 50 pixels each over the spectral ranges plotted in Figure 2. Without any interorder adjustment, the relative levels of the three shortest wavelength orders appear to match each other well in addition to being consistent with the $J$ and $H$ photometry.

In order to perform a check on the flux level of the $K$-band spectroscopy, the expected photometric flux in the $K$ band was calculated based on the timing of the spectroscopic observations over the known $K$-band variability represented in Figure 3. Taking $K_{\min }=17.37 \mathrm{mag}$ from Table 2 and adding to that the -0.70 mag difference between the average $K$-band minimum and maximum from Debes et al. (2006) yields $K_{\max }=16.67 \mathrm{mag}$.
From Figure 3, the spectroscopic observations span, over time, an estimated average minimum-to-maximum ratio of $5: 7$, yielding a flux-averaged magnitude of $K_{\mathrm{avg}}=17.02 \mathrm{mag}$ over all the exposures. All three of these $K$-band fluxes are plotted in Figure 2. As can be seen in the figure, the spectroscopic flux over the $K$ band is commensurate with expectations without any adjustments.

The spectrum in Figure 2 appears mostly consistent with a Rayleigh-Jeans slope in the $J$ band, where an emission line is likely detected at $\mathrm{Pa} \beta$ and possibly also at $\mathrm{Pa} \gamma$. It is unclear whether any emission originates on the companion via heating by the primary, or on the white dwarf via accretion; both are possible. A modest excess is detected over the $H$ band, and is consistent with the Table 2 photometry. At $K$ band there is a definite excess throughout the window and four possible noteworthy features with varying degrees of uncertainty: a slight peak near $2.1 \mu \mathrm{m}$ that could be a cyclotron harmonic, $\mathrm{Br} \gamma$ emission, the $\mathrm{CO}$ band head at $2.29 \mu \mathrm{m}$, as well as a significant upturn in slope near $2.3 \mu \mathrm{m}$.

\section{ANALYSIS}

\subsection{Binary Components}

The near-infrared spectrum of SDSS 1212 has been fitted with several spectral models and templates. The binary components were analyzed following the method of Dobbie et al. (2005). A model white dwarf spectrum spanning $0.3-10 \mu \mathrm{m}$ was generated for a nonmagnetic, pure hydrogen atmosphere at $T_{\text {eff }}=10,000 \mathrm{~K}$ and $\log g=8.0$ (Schmidt et al. 2005a), using tlusty ${ }^{5}$ (Hubeny $\&$ Lanz 1995) and synspec. ${ }^{6}$ Since the white dwarf has a significant magnetic field, with Zeeman-split Balmer lines in its optical spectrum, it is not possible at present to more precisely determine its parameters by the usual method of Balmer line modeling. The model spectrum has been normalized to the $g$ magnitude of the white dwarf and smoothed to match the spectral resolution of the GNIRS data.

Template $1-2.3 \mu \mathrm{m}$ spectra for brown dwarfs of late $\mathrm{L}$ and early T type were taken from low-resolution Keck NIRSPEC data sets (McLean et al. 2003). These templates were then extended to $2.5 \mu \mathrm{m}$ using similar near-infrared UKIRT CGS4 spectral data (Geballe et al. 2002; Leggett et al. 2001), in order that they span the full extent of the GNIRS wavelength coverage. The NIRSPEC spectral templates have similar resolution $(R \approx 2000)$ to the GNIRS data, and hence no smoothing of either data was performed. However, the CGS4 data had to be interpolated between resolution elements in order to create sufficient data points to compare with the GNIRS spectrum. Empirical model fluxes, determined from near-infrared photometry and parallaxes ( Tinney et al. 2003 ), were scaled appropriately to $d \approx 150 \mathrm{pc}$, the nominal distance to a white dwarf with the assumed parameters listed above.

\subsection{Cyclotron Emission}

A physically realistic model for cyclotron emission was calculated using the formulation and methods described in detail by Hoard et al. (2007a) and Brinkworth et al. (2007). The model employs five critical parameters to produce the emergent cyclotron spectrum. The magnetic field strength and electron temperature determine the cyclotron harmonic wavelengths, while the viewing angle affects the overall shape of the peaks. Also included are a parameter related to the transition from optically thick to optically thin emission (typically well constrained by the

\footnotetext{
5 See http://nova.astro.umd.edu.

6 See footnote 5.
} 
observations), and a scaling factor linked to the area of the emitting region: a few percent of the white dwarf surface area for the models shown here.

Initial attempts to fit the spectrum of SDSS 1212 using cyclotron emission in a $B=13 \mathrm{MG}$ (Schmidt et al. 2003) field were problematic. The sum of the white dwarf and secondary model components does a decent job of reproducing the phase-averaged spectrum shortward of $K$ band. Yet the addition of such a cyclotron model component in this bandpass does not account for the upturn near $2.3 \mu \mathrm{m}$ in the GNIRS data. Several circumbinary dust models were considered to explain this observed rise in flux at the end of the $K$ band, but were subsequently discarded on the realization that this feature must be a cyclotron harmonic for the following reasons.

As pointed out by Debes et al. (2006) the zero-temperature $m=4$ cyclotron harmonic for a $13 \mathrm{MG}$ magnetic field lies near $2.1 \mu \mathrm{m}$. But in such a model, this cyclotron feature alone, varied by $100 \%$, is insufficient to produce the peak-to-peak flux change ( $\Delta m \approx 1 \mathrm{mag}$ ) seen in their $K$-band light curve. Once the contributions of the white dwarf and secondary are accounted for, all the remaining flux in the $K$ band must be $100 \%$ variable in order to explain the observed light curve, leaving cyclotron as the only viable candidate for this extra emission. Furthermore, a $13 \mathrm{MG}$ magnetic field should produce cyclotron harmonics and photometric variability within the $H$ band ( $m=5$ would be near $1.6 \mu \mathrm{m})$, as seen in EF Eri and AM Her (Kafka et al. 2005; Harrison et al. 2004). The resolution to this dilemma is that the magnetic field of SDSS 1212 must be less than $13 \mathrm{MG}$, such that more cyclotron emission is present within the $K$ band, while the harmonics at shorter wavelengths are diminished.

A magnetic field of $7 \mathrm{MG}$ should have two zero-temperature cyclotron harmonics within the $K$ band, near 2.2 and $2.5 \mu \mathrm{m}$ for $m=6$ and 7, respectively. Although Schmidt et al. (2003) determine an equivalent dipolar field of $B_{d}=13 \mathrm{MG}$ for SDSS 1212 (via models and assumed geometry), the mean surface field as determined by the measured Zeeman splitting of $\mathrm{H} \alpha$ and $\mathrm{H} \beta$ is $B_{s}=7 \mathrm{MG}$ (Schmidt et al. 2005a). As shown below, this magnetic field strength is consistent with the absence of observable cyclotron harmonics in $H$ band, and the presence of two harmonics in the $K$ band, which together can explain the large photometric variability seen in this filter.

Figures 4 and 5 display the most successful model reproductions of the GNIRS cross-dispersed spectrum of SDSS 1212. The essential difference between the two figures is that an L8 dwarf template was used for the Figure 4 plots, while a T2 dwarf template was used in the plots of Figure 5. It should be noted that between these two figures, a minor scaling readjustment was made to the binary components in order to best reproduce the observed spectrum of SDSS 1212 . This rescaling amounted to $8 \%$, corresponding to a $4 \%$ change in the assumed distance to the system, well within the $14 \%$ minimum uncertainty arising from its poorly constrained temperature and unknown surface gravity (Burleigh et al. 2006a; Schmidt et al. 2005a).

The upper panels in both figures represent the best fit to the spectrum with only the binary components at $d \approx 150 \mathrm{pc}$ : the white dwarf model plus a brown dwarf template. Already it is clear that the $H$-band excess is largely reproduced by the addition of the L8 dwarf, but not as well by the T2 dwarf. However, in both upper panels there appears to be excess flux over the 1.05$1.20 \mu \mathrm{m}$ range compared to the model. It is not clear if this is the result of poor signal or calibration of order 6 of the crossdispersed GNIRS data, or a real detection.

The middle panels of both figures add a single field cyclotron emission component to the upper panel model fits. In both fig- ures, the models are for a 7.6 MG magnetic field, a $5 \mathrm{keV}$ plasma temperature, and a viewing angle of $75^{\circ}$, with the remaining parameters varied slightly so that the peaks of the cyclotron harmonics match the phase-averaged $K$-band flux. Although this set of models can account for a significant portion of the $K$-band flux and hence go a long way toward accounting for the photometric variability in this window, they fail miserably in the region between the cyclotron peaks. It is perhaps noteworthy that the T2 model requires a stronger cyclotron component than does the L8 model (due to the bluer $H-K$ color of T dwarfs vs. L dwarfs) and is more readily consistent with the $K$-band variability.

The lower panels in both figures display cyclotron models similar to the middle panels, but with a viewing angle of $50^{\circ}$, a value commensurate with the determination by Burleigh et al. (2006a). Although this angle is not strongly constrained by their data, the GNIRS spectra appear to demand an intermediate viewing angle, whose result is to broaden the cyclotron harmonics, effectively blurring them together for low viewing angles (Hoard et al. 2007a). In Figure 4 the magnetic field employed is $7.0 \mathrm{MG}$, while in Figure 5 it is $7.2 \mathrm{MG}$, both adjusted slightly to best fit the overall spectrum when combined with their respective secondary models. Both these model fits do a decent job of reproducing the entire spectrum from 1.2 to $2.5 \mu \mathrm{m}$, but the $\mathrm{L} 8$ model matches the $H$ - and $K$-band data more closely than the T2 model, as in the middle panels.

\subsection{Substellar Secondary}

The three-component, composite model fits contain a sufficient number of free parameters that their success in reproducing the $1.2-2.5 \mu \mathrm{m}$ spectrum of SDSS 1212 may not seem surprising, but is nonetheless informative. During initial attempts to model the GNIRS spectrum, secondary spectral templates of types L6 and T5 were shown to be significantly too bright and too dim, respectively, beyond $1.5 \mu \mathrm{m}$ when their overall $J$-band flux level was matched to the data. Figure 4 is consistent with the direct spectroscopic detection of a substellar object with spectral type L8. While the T2 model in Figure 5 appears somewhat inconsistent with the data, it does not rule out an earlier type T dwarf, for which available templates were lacking. Hence, the companion to SDSS 1212 has a likely spectral type between L8 and T1. Uncertainty in the distance to the white dwarf allows for some leeway in the relative contribution of the binary components at near-infrared wavelengths. While its poorly constrained effective temperature permits photometric distances both nearer or farther than $d \approx 150 \mathrm{pc}$, the unconstrained mass and radius of the white dwarf are likely to overpredict its distance. A typical isolated white dwarf surface gravity, or $\log g=8.0\left(0.60 M_{\odot}\right.$ at $T_{\text {eff }}=10,000 \mathrm{~K}$; Bergeron et al. 1995), is more likely to be too low than too high, especially if the primary has been accreting material over gigayear timescales. This would tend to reduce the photometric distance and lead to later spectral types for the companion. If SDSS 1212 is more massive than $\log g=8.0$, there is a greater likelihood that the companion has an early $\mathrm{T}$ spectral type. The lower panel in the L8 model, which also matches the observed $K$-band spectrum well, seems to indicate that the $\mathrm{CO}$ band head at $2.29 \mu \mathrm{m}$ may have been detected, albeit just barely. If correct, it would be further evidence that the companion is a late L dwarf, since this $\mathrm{CO}$ feature weakens significantly in T dwarfs (Cushing et al. 2005; McLean et al. 2003; Geballe et al. 2002).

\subsection{The Nature of SDSS 1212}

The two models for the nature of mass transfer and accretion in SDSS 1212 are Roche lobe overflow and wind capture. The 

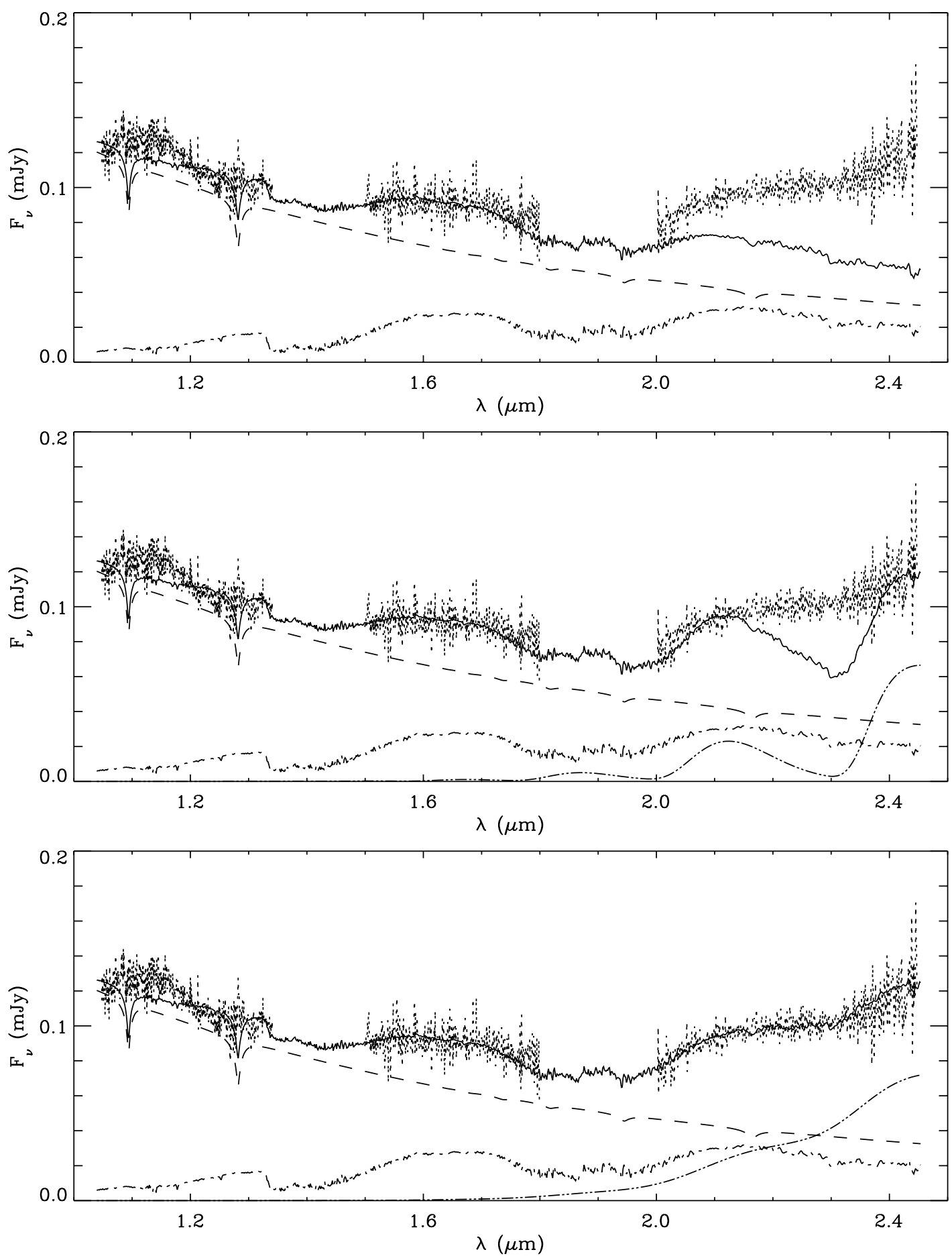

FIG. 4.- Model fits to the GNIRS, phase-averaged, near-infrared spectrum of SDSS 1212. In each panel the data are represented by a dotted line, and the fit is shown as a solid line. Some rescaling has taken place between panels to best fit the data, but the binary components remain at $d \approx 150$ pc in all plots. Details of the models and fits are discussed in $\delta \S 4.1$ and 4.2. Top: The dashed line is a pure hydrogen atmosphere, $T_{\text {eff }}=10,000 \mathrm{~K}$ model for a $0.6 M \odot$ white dwarf. The dash-dotted line is an L8 brown dwarf template spectrum. Middle: The double-dash-dotted line shows the cyclotron model at a viewing angle of $75^{\circ}$. Bottom: Here, the double-dash-dotted line shows the cyclotron model at a viewing angle of $50^{\circ}$.

near-infrared observations do not distinguish between these two evolutionary scenarios, but are informative for the following reason. The confirmed presence of cyclotron radiation adds one more observational property that SDSS 1212 now shares with polars (especially those with likely substellar secondaries in persistent low states such as EF Eri). The traits it shares are: an appreciable $\mathrm{X}$-ray luminosity, the presence of an accretion hot spot, a vari- able optical light curve with a similar shape to those of known polars in low states (i.e., with larger modulations in the blue and ultraviolet compared to the red), a spin-locked binary configuration, and cyclotron emission (Burleigh et al. 2006a; Schmidt et al. 2005a). Occam's razor would argue that SDSS 1212 is a polar.

Furthermore, the hot spot X-ray luminosity of SDSS 1212 is $L_{\mathrm{X}} \approx 3 \times 10^{29} \mathrm{ergs} \mathrm{s}^{-1}$, which is nearly identical to that measured 

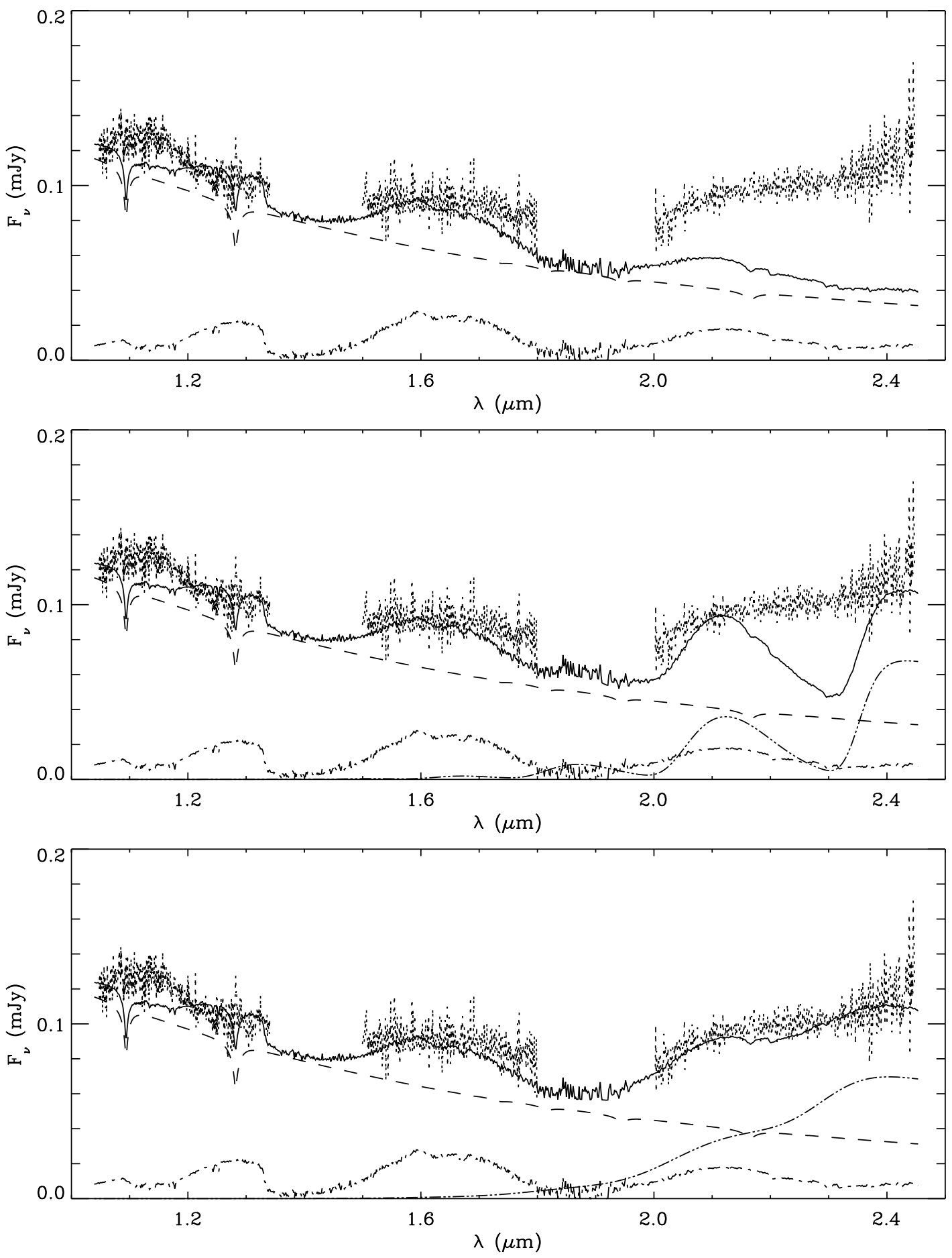

FIG. 5.- Same as Fig. 4, but for a T2 brown dwarf template spectrum.

for EF Eri during its $10 \mathrm{yr}$ low: namely, $L_{\mathrm{X}} \approx 2 \times 10^{29} \mathrm{ergs} \mathrm{s}^{-1}$ (Schwope et al. 2007). While the X-ray emission from many suspected, detached, wind capture systems may be too weak (and more importantly, not periodically variable) to originate via accretion onto the white dwarf, but rather from activity on the $\mathrm{M}$ dwarf secondary, $\mathrm{L}$ dwarfs simply do not have sufficient X-ray luminosities, hence favoring the low-state polar model for SDSS 1212 (Burleigh et al. 2006a). Also, there is the issue of how such a low-mass object generates sufficient wind to be captured by the magnetic white dwarf. To date, no model has emerged which can explain how an old $\mathrm{L}$ or $\mathrm{T}$ dwarf might generate a wind of order $10^{-13} M_{\odot} \mathrm{yr}^{-1}$.

\section{DISCUSSION}

\subsection{Past and Future Evolution}

The gross evolution cataclysmic variables should consist of three fundamental phases:

1. A short- to long-lived phase as a detached main-sequence binary which subsequently evolves to shorter orbital periods through 
common-envelope evolution as the primary leaves the main sequence and becomes a first ascent or asymptotic giant. Angular momentum is transferred, via friction, into the envelope itself, ejecting it in the process (Paczynski 1976). If the orbit is still too large for the secondary to make contact with its Roche lobe, then three mechanisms can bring this about, in principle: gravitational radiation, magnetic braking, or nuclear evolution. The timescales of these processes depend on the masses involved, but if one assumes a canonical $0.6 M_{\odot}$ white dwarf and a $0.2 M_{\odot}$ secondary (corresponding to the mass distribution peak among detached, unevolved, low-mass companions to white dwarfs; Farihi et al. $2005 \mathrm{a}$ ), then both gravitational radiation and magnetic braking operate within gigayear timescales for period ranges $P \lesssim 3$ and $P>3 \mathrm{hr}$, respectively, whereas nuclear evolution will play no role within this time frame (Patterson 1984).

2. A phase in which the shrinking Roche lobe of the secondary becomes smaller than its radius, causing unstable mass transfer onto the white dwarf. In principle this is a runaway inspiral until the mass ratio is close to 1 , but if the secondary has $M<$ $0.5 M_{\odot}$ to begin with (as it should in the vast majority of cases based on the aforementioned companion mass distribution), then conservation of angular momentum competes to partially counterbalance the losses due to any braking (magnetic or tidal) and gravitational radiation (Nelemans et al. 2001). At some point the trend reverses outward as the orbital evolution becomes dominated by conservation of angular momentum and any mass lost from the system entirely (e.g., nova-like outbursts, accretiondriven winds).

3. A final, debatable, phase consisting of a once more detached or very low accretion rate semidetached binary comprised of two degenerate objects in an expanded orbit which persists (now subject only to gravitational radiation) up to 10 Gyr or so, depending on the final masses and separation. This eventual state depends on the thermal and nuclear history of the secondary, but requires a degenerate secondary core in order to cease expansion permanently. Thus, a system may end its existence as a white dwarf with a close, altered substellar companion, both objects cooling gradually toward oblivion.

The ultimate fate of cataclysmic variables outlined above is uncertain for two fundamentally different reasons. The first issue is that the outcome itself is unknown, with end predictions for secondaries including: complete stripping, tidal disruption, persistence as semidetached substellar donors with very low mass transfer rates, and survival as detached substellar or even planetary-mass objects (Littlefair et al. 2003; Kolb \& Baraffe 1999; Patterson 1998; Howell et al. 1997). The second issue is detectability, which itself divides into two categories: searching for either erupting systems or their burnt-out remnants.

\subsection{Recently Deceased or Born Dead?}

So far, astronomers who study cataclysmic variables have concentrated primarily on those systems known to be eruptive in order to search for and understand possible end states, including substellar companions. The means of identifying such low-mass secondary systems relies heavily on semiempirical and theoretical relations between observed periods and spectral type, mass ratio, effective temperature, and mass transfer rate (Littlefair et al. 2006, 2007; Harrison et al. 2005; Patterson et al. 2005; Kolb \& Baraffe 1999; Howell et al. 1997). Without models, there is no means of determining whether a given substellar companion is migrating inward or outward, i.e., approaching or leaving its period minimum. With the prior history of these interacting systems unknown, the beginning and end states may appear identical, theoretically.
TABLE 3

Close Binary Parameters for SDSS 1212 and WD 0137-349

\begin{tabular}{crccccc}
\hline \hline Star & $\begin{array}{c}P \\
(\mathrm{~min})\end{array}$ & $\begin{array}{c}M_{1} \\
\left(M_{\odot}\right)\end{array}$ & $\begin{array}{c}M_{2} \\
\left(M_{\odot}\right)\end{array}$ & $\begin{array}{c}a \\
\left(R_{\odot}\right)\end{array}$ & $\begin{array}{c}R_{2} \\
\left(R_{\odot}\right)\end{array}$ & $\begin{array}{c}R_{L 2} \\
\left(R_{\odot}\right)\end{array}$ \\
\hline WD 0137-349 ……......... & 115.6 & 0.39 & 0.05 & 0.60 & 0.09 & 0.13 \\
SDSS 1212.................. & 88.4 & 0.60 & 0.05 & 0.57 & 0.09 & 0.11 \\
\hline
\end{tabular}

Notes.-Values for the white dwarf and secondary masses in the SDSS 1212 system are assumed, since no measurements exist. However, the resulting semimajor axis, unperturbed secondary radius, and secondary Roche lobe radius are all relatively insensitive to those input values, and reflect likely parameters. The semimajor axis for WD 0137-349 was calculated using Kepler's law, yielding a value which differs slightly from that reported in Maxted et al. (2006).

In addition, it is difficult to know with certainty what the size of the secondary star is in such systems, especially if the mass transfer rate is low - consistent with quiescence or an underfilled Roche lobe (i.e., a noncataclysmic variable). If a companion does indeed have a radius smaller than its Roche lobe, then strictly speaking it is detached, and the question remains: Is Roche lobe contact in its future or in its past? The recently identified lowmass transfer magnetic binaries reported in Schmidt et al. (2005b) are good candidates for being detached due to the combination of the inferred accretion rates, orbital periods, and extremely low or absent X-ray emission. But if EF Eri can turn off for nearly $10 y$ r, then so can other polars (Schwope et al. 2007). SDSS 1212 sits somewhere on the border between a typical polar and the low accretion rate systems, having respectable X-ray accretion luminosity and a relatively low inferred mass transfer rate (Burleigh et al. 2006a). If one assumes the substellar companion to SDSS 1212 has a mass and effective temperature similar to WD $0137-$ 349B (L8 V; see Table 3; Burleigh et al. 2006b), then its unperturbed radius should be $0.09 R_{\odot}$ for ages between 1 and $5 \mathrm{Gyr}$ (Baraffe et al. 2003), but its Roche lobe should be located roughly at $0.11 R_{\odot}$ for a $0.60 M_{\odot}$ primary. Therefore, the companion is either: (1) recently deceased, far out of thermal equilibrium and inflated as expected for an object past period minimum with the implied long history of mass-radius imbalance; or (2) born dead, intrinsically detached, and slamming the magnetic white dwarf with all of its (irradiatively driven?) wind. This raises similar questions about the nature of all other low accretion rate magnetic binaries.

Depending on the past thermal and nuclear history of the secondary, especially if the core is degenerate, and how much mass escapes the binary completely and adiabatically, it is quite possible for the orbital period to increase significantly over the minimum into a region where no interaction (or wind accretion only) occurs (Howell et al. 1997; Jeans 1924). Without any distinct spectral features or mass-radius anomalies in such detached binaries, there may be little or no means to differentiate empirically between the pre- and post- states of these innately interacting systems.

\subsection{The Frequency of the Doubly Dead}

Objects must first be detected as a cataclysmic variable in order to look for substellar companions; hence, selection effects play a large role in the number of systems suspected to harbor such cool degenerate companions (Littlefair et al. 2003). However, this overlooks the obvious question: If the end states are detached double degenerate systems, where are they? If one restricts a search to eruptive systems, then the potential for success is limited from the beginning for the reasons stated above. Why not search directly for systems which are detached and which 
may represent final states? This seems to be the only way to reconcile the well-known discrepancy between the number of predicted objects past period bounce and the number observed or suspected from observation (Patterson 1998; Howell et al. 1997).

Clearly, the best place to look for these types of end states is in the infrared, where cool, Jupiter-sized companions easily outshine their tiny white dwarf primaries (Farihi et al. 2005a, 2005b). There are only two issues associated with a search around white dwarfs for extinct cataclysmics - the white dwarf must first have been identified or previously known, and one must have sufficient sensitivity to detect its cooling leftovers. If the end state white dwarfs are very cool and far away such that $V>21 \mathrm{mag}$ (e.g., $T_{\text {eff }}<5000 \mathrm{~K}$ and $d>100 \mathrm{pc}$ for $M=0.9 M_{\odot}$ ), then only the Sloan Digital Sky Survey will detect them, but will be quite insensitive to any substellar companions. While the above conditions may not be representative, given that cataclysmic variables appear to be roughly 100 times less abundant than white dwarfs (Patterson 1984), a large sample of targets would be required to perform a statistically rigorous search; if all cataclysmic variables are in this state, a search would only yield 1 per 100 white dwarfs (not very encouraging).

If these close but detached systems exist in any significant numbers, they have not yet been identified from various surveys of relatively nearby white dwarfs. Spatially unresolved L-type companions, corresponding to $0.05-0.07 M_{\odot}$ for unevolved ages of 1-5 Gyr, are rare, with only three known among well over $N=1000$ targets (Hoard et al. 2007b; Burleigh et al. 2006b; Farihi et al. 2005a; Farihi 2004; Farihi \& Christopher 2004; Wachter et al. 2003). Spatially unresolved T-type companions, corresponding to $0.02-0.05 M_{\odot}$ for unevolved ages of 1-5 Gyr, are also rare, with none detected or suspected in various surveys totalling $N \approx 200$ targets (Mullaly et al. 2007; Hansen et al. 2006; Farihi et al. 2005a; J. Farihi, E. E. Becklin, \& B. Zuckerman 2008 , in preparation). It is possible that stellar evolution has yet to produce such systems; i.e., the initial mass function for secondaries in such systems, convolved with the finite age of the Galaxy, implies that the vast majority of these systems will continue in phases (1) and (2) for up to $10 \mathrm{Gyr}$. It could be that the lack of detected end states is an observational bias; if these systems are still marginally semidetached, then the signature of the secondary star could be masked by the accretion disk in dwarf novae and by cyclotron emission in polars. Alternatively, phase (3) never occurs, and the companion is cannibalized, evaporated, stripped, or tidally destroyed. This would also explain the lack of observed end states.

For completeness, something ought to be said about the empirical frequency of zero-age cataclysmic variables with a brown dwarf secondary (Politano 2004). Since their pre- states would consist of a white dwarf with a detached substellar companion, the above arguments and evidence apply $(f<0.5 \%)$. In addition, the progenitors of such pre- states would be main-sequence stars with brown dwarf companions in the few AU range, which are also extremely rare, occurring with a well-constrained frequency, $f<0.5 \%$ (Butler et al. 2006; Marcy et al. 2005). Hence, there should be no doubt that cataclysmic variables form with brown dwarf secondaries quite rarely.

\subsection{Detached Companions to Magnetic White Dwarfs}

The same argument which applies to detached substellar companions to white dwarfs also applies to detached stellar companions to magnetic white dwarfs, although in this latter case finding them should be easier in principle (Liebert et al. 2005). One might suggest a sensitive search for magnetism among known lists of close but detached, and wide common propermotion companions. Large lists of these white dwarf plus red dwarf systems exist in the following references: for known radial velocity pairs, see Morales-Rueda et al. (2005); for spatially unresolved pairs, see Hoard et al. (2007b), Farihi et al. (2005a, 2006), and Wachter et al. (2003); and for wide common propermotion pairs, see Farihi et al. (2005a) and Silvestri et al. (2005). All of these lists contain targets which are likely to be superior to white dwarfs from the Sloan Digital Sky Survey, since they are brighter and, hence, more sensitive searches could be conducted. However, it should be noted that the main observational indicators of a magnetic white dwarf, Zeeman splitting and circular spectropolarimetry, are strongest in $\mathrm{H} \alpha$ and $\mathrm{H} \beta$. Unfortunately, in unresolved white dwarf plus red dwarf binaries (i.e., the potential cataclysmic variables) these features, especially $\mathrm{H} \alpha$, are often completely filled in by the red dwarf. This may be another reason for the curious lack of detected prepolar systems to be considered along with those already discussed by Liebert et al. (2005).

\section{FUTURE WORK}

Unfortunately, the intrinsic faintness of SDSS 1212 precludes several interesting avenues of follow-up, such as phase-resolved near-infrared spectroscopy or mid-infrared spectroscopy. Ultraviolet and X-ray observations are currently planned to firmly distinguish between standard mass transfer through Roche lobe overflow versus accreted wind. High-resolution optical spectra of SDSS 1212 may reveal the presence of elements accreted from its companion. Such observations could, in principle, distinguish between Roche lobe overflow mass transfer and wind accretion as well.

There may be little or no hope of directly detecting the bare substellar companion beyond the $H$-band spectrum presented here. The 7 MG cyclotron models used successfully in this paper predict the fundamental harmonic wavelength should be $15.3 \mu \mathrm{m}$, thus potentially swamping every instrument aboard Spitzer which might otherwise be capable of directly detecting the flux of an L8 dwarf at $d=150 \mathrm{pc}$ : namely, IRAC 3-8 $\mu$ m photometry, and IRS $16 \mu$ m peak-up imaging. However, IRAC observations should directly test the lower magnetic field cyclotron models employed to fit the GNIRS data, which predict relatively strong emission near 3.1, 3.8, 5.1, and 7.7 $\mu \mathrm{m}$, corresponding to the zerotemperature $m=5,4,3$, and 2 harmonic numbers in a $7.0 \mathrm{MG}$ magnetic field. Yet the possible presence of circumbinary dust emitting at IRAC wavelengths, as seen in several polars (Hoard et al. 2007a; Brinkworth et al. 2007; Howell et al. 2006), may complicate the interpretation of any mid-infrared photometry for SDSS 1212.

This paper benefitted greatly from the insight of an anonymous referee, to whom the authors are commensurately grateful. The authors would like to thank the following people: B. Rodgers, G. Doppman, and K. Labrie (for much appreciated help in understanding and processing GNIRS data); A. Stephens (for NIRI particulars); P. Hirst (for WFCAM particulars); and S. Leggett (for a useful discussion regarding photometric calibration errors). M. R. Burleigh is supported by a UK Science and Technology Facilities Council Advanced Fellowship. Spectroscopic observations for this paper were taken as part of the Gemini Director's Discretionary Time GS-2006A-DD-5. Photometric observations were obtained as part of Gemini Queue Program GN-2006A-Q-51 and UKIRT Service Program 1653. The Gemini Observatory 
is operated by the Association of Universities for Research in Astronomy, Inc., under a cooperative agreement with the NSF on behalf of the Gemini partnership: the National Science Foundation (United States), the Particle Physics and Astronomy Research Council (United Kingdom), the National Research Council (Canada), CONICYT (Chile), the Australian Research Council (Australia), CNPq (Brazil), and CONICET (Argentina). The United Kingdom Infrared Telescope is operated by the Joint Astronomy Centre on behalf of the UK Particle Physics and Astronomy
Research Council. This publication makes use of data products from the Two Micron All Sky Survey, which is a joint project of the University of Massachusetts and the Infrared Processing and Analysis Center/California Institute of Technology, funded by the National Aeronautics and Space Administration and the National Science Foundation. This paper has made use of the Aladin Sky Atlas operated at CDS, Strasbourg, France.

Facilities: Gemini:Gillett, Gemini:South, UKIRT (WFCAM)
Baraffe, I., Chabrier, G., Barman, T. S., Allard, F., \& Hauschildt, P. H. 2003, A\&A, 402, 701

Bergeron, P., Saumon, D., \& Wesemael, F. 1995, ApJ, 443, 764

Brinkworth, C. S., et al. 2007, ApJ, 659, 1541

Burleigh, M. R., Hogan, E. M., Dobbie, P. D., Napiwotzki, R., \& Maxted, P. F. L. 2006a, MNRAS, 373, L55

Burleigh, M. R., et al. 2006b, MNRAS, 373, 1416

Butler, R. P., et al. 2006, ApJ, 646, 505

Carpenter, J. M. 2001, AJ, 121, 2851

Cushing, M. C., Rayner, J. T., \& Vacca, W. D. 2005, ApJ, 623, 1115

Cushing, M. C., Vacca, W. D., \& Rayner, J. T. 2004, PASP, 116, 362

Debes, J. H., López-Morales, M., Bonanos, A. Z., \& Weinberger, A. J. 2006, ApJ, 647, L147

Dobbie, P. D., Burleigh, M. R., Levan, A. J., Barstow, M. A., Napiwotzki, R., Holberg, J. B., Hubeny, I., \& Howell, S. B. 2005, MNRAS, 357, 1049

Elias, J. H., et al. 1998, Proc. SPIE, 3354, 555

Farihi, J. 2004, Ph.D. thesis, Univ. California, Los Angeles

Farihi, J., Becklin, E. E., \& Zuckerman, B. 2005a, ApJS, 161, 394

Farihi, J., \& Christopher, M. 2004, AJ, 128, 1868

Farihi, J., Hoard, D. W., \& Wachter, S. 2006, ApJ, 646, 480

Farihi, J., Zuckerman, B., \& Becklin, E. E. 2005b, AJ, 130, 2237

Geballe, T. R., et al. 2002, ApJ, 564, 466

Hansen, B. M. S., Kulkarni, S., \& Wiktorowicz, S. 2006, AJ, 131, 1106

Harrison, T. E., Howell, S. B., Szkody, P., \& Cordova, F. A. 2005, ApJ, 632, L123

Harrison, T. E., Howell, S. B., Szkody, P., Homeier, D., Johnson, J. J., \& Osborne, H. L. 2004, ApJ, 614, 947

Hawarden, T. G., Leggett, S. K., Letawsky, M. B., Ballantyne, D. R., \& Casali, M. M. 2001, MNRAS, 325, 563

Henry, D. M., et al. 2003, Proc. SPIE, 4841, 63

Hirst, P., Casali, M. M., Adamson, A., Ives, D., \& Kerr, T. 2006, Proc. SPIE, 6269,31

Hoard, D. W., Howell, S. B., Brinkworth, C. S., Ciardi, D. R., \& Wachter, S. W. 2007a, ApJ, 671, 734

Hoard, D. W., Wachter, S., Sturch, L. K., Widhalm, A. M., Weiler, K. P., Pretorius, M. L., Wellhouse, J. W., \& Gibiansky, M. 2007b, AJ, 134, 26

Hodapp, K. W., et al. 2003, PASP, 115, 1388

Howell, S. B., Rappaport, S., \& Politano, M. 1997, MNRAS, 287, 929

Howell, S. B., et al. 2006, ApJ, 646, L65

Hubeny, I., \& Lanz, T. 1995, ApJ, 439, 875

Jeans, J. H. 1924, MNRAS, 85, 2

\section{EFERENCES}

Kafka, S., Honeycutt, R. K., Howell, S. B., \& Harrison, T. E. 2005, AJ, 130, 2852

Koen, C., \& Maxted, P. F. L. 2006, MNRAS, 371, 1675

Kolb, U., \& Baraffe, I. 1999, MNRAS, 309, 1034

Leggett, S. K., Allard, F., Geballe, T. R., Hauschildt, P. H., \& Schwietzer, A. 2001, ApJ, 548, 908

Liebert, J., et al. 2005, AJ, 129, 2376

Littlefair, S. P., Dhillon, V. S., Marsh, T. R., Gänsicke, B. T., Baraffe, I., \& Watson, C. A. 2007, MNRAS, 381, 827

Littlefair, S. P., Dhillon, V. S., Marsh, T. R., Gänsicke, B. T., Southworth, J., \& Watson, C. A. 2006, Science, 314, 1578

Littlefair, S. P., Dhillon, V. S., \& Martín, E. L. 2003, MNRAS, 340, 264

Marcy, G. W., Butler, R. P., Fischer, D., Vogt, S. S., Wright, J. T., Tinney, C. G., \& Jones, H. R. A. 2005, Prog. Theor. Phys. Suppl., 158, 24

Maxted, P. F. L., Napiwotzki, R., Dobbie, P. D., \& Burleigh, M. R. 2006, Nature, 442, 543

McLean, I. S., McGovern, M. R., Burgasser, A. J., Kirkpatrick, J. D., Prato, L., \& Kim, S. S. 2003, ApJ, 596, 561

Morales-Rueda, L., Marsh, T. R., Maxted, P. F. L., Nelemans, G., Karl, C., Napiwotzki, R., \& Moran, C. K. J. 2005, MNRAS, 359, 648

Mullaly, F., Kilic, M., Reach, W. T., Kuchner, M. J., von Hippel, T., Burrows, A., \& Winget, D. E. 2007, ApJS, 171, 206

Nelemans, G., Yungelson, L. R., Portegies Zwart, S. F., \& Verbunt, F. 2001, A\&A, 365, 491

Paczynski, B. 1976, in IAU Symp. 73, Structure and Evolution of Close Binary Systems, ed. P. Eggleton, S. Mitton, \& J. Whelan (Dordrecht: Reidel), 75 Patterson, J. 1984, ApJS, 54, 443 . 1998, PASP, 110, 1132

Patterson, J., Thorstensen, J. R., \& Kemp, J. 2005, PASP, 117, 427

Politano, M. 2004, ApJ, 604, 817

Schmidt, G. D., Szkody, P., Silvestri, N. M., Cushing, M. C., Liebert, J. L., \&

Smith, P. S. 2005a, ApJ, 630, L173

Schmidt, G. D., et al. 2003, ApJ, 595, 1101

2005b, ApJ, 630, 1037

Schwope, A. D., Staude, A., Koester, D., \& Vogel, J. 2007, A\&A, 469, 1027 Silvestri, N. M., Hawley, S. L., \& Oswalt, T. D. 2005, AJ, 129, 2428 Skrutskie, M. F., et al. 2006, AJ, 131, 1163

Tinney, C. G., Burgasser, A. J., \& Kirkpatrick, J. D. 2003, AJ, 126, 975

Vacca, W. D., Cushing, M. C., \& Rayner, J. T. 2003, PASP, 115, 389

Wachter, S., Hoard, D. W., Hansen, K. H., Wilcox, R. E., Taylor, H. M., \& Finkelstein, S. L. 2003, ApJ, 586, 1356 